\title{
Structural Motion Monitoring Using 9-Axis Sensing Modules with Wireless Communication Capability
}

\author{
Hou Xiaoyan*, Kuang KSC \\ Department of Civil and Environmental Engineering, National University of Singapore, Singapore
}

Received: October 13, 2016; Accepted: October 17, 2016; Published: November 08, 2016

*Corresponding author: Hou Xiaoyan, Department of Civil and Environmental Engineering, National University of Singapore, Singapore, Email: houxiaoyan@gmail.com

\begin{abstract}
Dynamic motion monitoring is useful for many applications such as modal testing, mechanical design, vibration control, structural coupling and theoretical model modifications. New sensing systems and sensing techniques for both translational and rotational motion are always in great need. This paper present two compact and portable motion sensing systems to monitor the dynamic behavior of complicated structures. Each system consists of a 9-axis sensing module and a control computer.

As the sensing modules implement signal filtering, analogue to digital conversion and motion analysis on chip, no additional signal amplifiers are necessary. The modules get their power supply from the USB (Universal Serial Bus) port of the computer without involvement of extra power unit. The same cable for the sensor power supply serves to transfer the measured data from the module to the computer thus no oscilloscope is needed. The elimination of sensor power unit, amplifiers and oscilloscope greatly simplifies the measurement system and makes on-site motion monitoring much more convenient, especially when the accessible space is limited. The inertial loading and local stiffening effects introduced by conventional sensors are also reduced. In addition, for cases where the cable connection between the module and the computer is difficult or even impossible, wireless transmission modules can be incorporated into the system to form a wireless measurement system. Through experiments, it is found that the wireless data maintain their quality even after passing through a few concrete walls when wireless transmission distance is more than 60 meters.
\end{abstract}

Keywords: Translational Motion Measurement; Rotational Motion Measurement; Wireless Sensor Network; Structural Health Monitoring JY-901 9-Axis Module; GY953 9-Axis Module;

\section{Introduction}

Structures are subject to harsh environments in their life cycles from extreme and cyclical loading conditions, and possibly from internal and external corrosion (Zhu et al. 2016). These present significant challenges to the structure systems, which are used to serve for years and endure risk of failure (Frangopol et al. 2015). The structural failures such as overload, jamming and parts damage will result in serious losses of properties, ecocatastrophes and even deaths (Brownjohn et al. 2011). All these are difficult to be identified by periodical inspections or routine safety operations.

As an example, operating vessels at sea is a highly challenging activity by its nature (Kongsberg Maritime 2016). In order to undertake tasks such as wave height monitoring or using cranes during rough weather, roll, pitch, yaw motion needs to be measured. Traditional sensors like Motion Reference Units (MRUs) though can provide accurate measurement; they are complex, expensive and big in size. New sensors or sensing systems with high reliability and low cost are in great demand for large-scale industrial deployment.

Traditional IMUs like Honeywell and Sagem are used in many applications for navigation, control and human environment interaction (Yuan et al. 2014, Ren et al. 2014). With AHRS (attitude and heading reference system), it is possible to get similar performance in a smaller package which can caters for more applications, such as antenna and camera stabilization, small unmanned vehicles and even hand-held applications.

In this paper, structural motion measurement systems based on 9-Axis MEMS (Micro Electro Mechanical Sensor) sensing modules are developed. Attitude sensing modules JY-901 and GY953 are used in monitoring the translational acceleration and rotational motion of structures. Wireless transmission modules may be incorporated into the measurement system when the cable connection between the module and the computer is difficult or even impossible. The measured motion data give us insight into how the structures work and provide useful information for the design optimization of the structures.

Accurately tracking complex motion requires the use of motion sensors such as gyroscopes, accelerometers, compasses and pressure sensors. The collected sensor data are then fused into a single and accurate data stream for use as input commands in electronics devices. The JY-901 module combines acceleration and rotational motion plus heading information into a single data stream for applications (Junyue 2015). It has a small footprint and cost advantages compared to discrete gyroscope plus accelerometer solutions. It is capable to interface with multiple non-inertial digital sensors, such as pressure sensors, on its auxiliary I2C port.

The JY-901 collects gyroscope, accelerometer and magnetometer data while synchronizing data sampling at a user- 
defined rate. Internal Digital Motion Processing (DMP) engine supports three dimensional motion processing. The FIFO (First In, First Out) buffers the complete data set and reduces timing requirements on the system processor. After burst reading the FIFO data, the system processor can save power by entering a low-power sleep mode while collecting more data.

Compared with JY-901, GY953 is even smaller yet also powerful. GY953 is AHRS electronic compass module with tilting compensation. Affordable MEMS AHRS systems were introduced just after 2000, when inertial sensor price dropped and accuracy improved. The improved inertial sensors were used by companies such as Xsens to design AHRS that provide three dimensional orientations for a broad range of applications from human motion to navigation of unmanned vehicles and the stabilization of antenna and camera systems.

\section{JY-901 9-Axis Sensing Module}

The JY-901 attitude sensing module (Figure1) employs MPU6050 as its motion sensing and processing unit. MPU-6050 is one of the world's first few motion processing solutions with an integrated 9-axis sensor for applications like handset and tablet applications, game controllers, remote controls, and other consumer devices. The MPU-6050 has an embedded 3-axis MEMS gyroscope, a 3-axis MEMS accelerometer, and a Digital Motion Processor (DMP). When connected to a 3-axis magnetometer, the MPU-6050 delivers a complete 9-axis Motion Fusion output to its primary I2C port.

\section{JY-901 Technical Specifications}

For attitude angles measurement, the attitude sensing module of the JY-901 has three independent vibratory MEMS gyroscopes, which detect rotation about the $\mathrm{X}, \mathrm{Y}$ and $\mathrm{Z}$ Axes respectively. When the gyros are rotated about any of the axes, the Coriolis Effect causes a vibration which is detected by a capacitive pickoff. The resulting signal is amplified, demodulated, and filtered to produce a voltage that is proportional to the angular rate. This voltage is digitized using the individual on-chip 16-bit Analog-toDigital Converters (ADCs) to sample each axis.

The full-scale range of the gyro sensors may be digitally programmed to $\pm 250, \pm 500, \pm 1000$, or \pm 2000 degrees per second

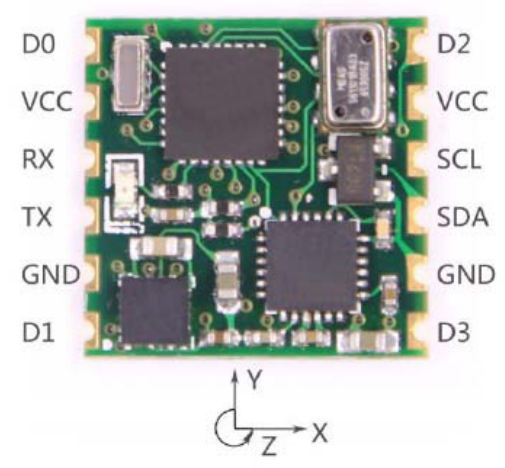

Figure 1: JY-901 attitude sensing module (dps). The ADC sample rate is programmable from 8,000 samples per second down to 3.9 samples per second. User-selectable low-pass filters enable a wide range of cut-off frequencies from $5 \mathrm{~Hz}$ to $256 \mathrm{~Hz}$. Orientation errors of the gyroscope mounted to the printed circuit board usually causes the cross-axis sensitivity in which one gyro or accelerometer responds to rotation or acceleration about another axis. For example, the $\mathrm{X}$ axis gyroscope may respond to a rotation about the $\mathrm{Y}$ or $\mathrm{Z}$ axis. In the JY-901, the cross-axis sensitivity of each gyro scope is $2 \%$. The resonant frequency in $\mathrm{X}, \mathrm{Y}$ and $\mathrm{Z}$ axis is about $33 \mathrm{KHz}$, far above the natural frequencies of the target test structures.

For acceleration measurement, the 3-axis accelerometer in the JY-901uses separate proof masses for each axis to realize small cross-sensitivity of $2 \%$. External acceleration along a particular axis induces displacement on the corresponding proof mass. Then the corresponding capacitive sensor detects the displacement differentially. The module features three 16-bit ADCs for digitizing the accelerometer outputs and each sensor has a dedicated sigma-delta ADC. For precious tracking of both fast and slow motions, the full scale range can be adjusted to $\pm 2 \mathrm{~g}$, $\pm 4 \mathrm{~g}, \pm 8 \mathrm{~g}$ or $\pm 16 \mathrm{~g}$. When the device is placed on a flat surface, it measures $0 \mathrm{~g}$ on the $\mathrm{X}$ and $\mathrm{Y}$ axes and $+1 \mathrm{~g}$ on the $\mathrm{Z}$ axis. The accelerometers' scale factor is nominally independent of supply voltage.

The JY-901 module supports a variety of advanced motionbased applications on-chip. In this way, it enables low-power motion processing in portable applications with reduced processing requirements for the system processor. In JY901, the DMP acquires data from accelerometers, gyroscopes, and additional $3^{\text {rd }}$ party sensors such as magnetometers, and processes the data. The resulting data can be read from the DMP's registers or buffered in a FIFO. In order to provide accurate results with low latency, motion processing algorithms are required to be run at a rate around $200 \mathrm{~Hz}$. This is necessary even when the application updates at a much lower rate such as $5 \mathrm{~Hz}$.

The JY-901 supports I2C communications on both its primary serial interface and its auxiliary interface. The auxiliary I2C bus is to communicate with the off-chip 3-axis digital magnetometer or other sensors. The auxiliary bus has two operating modes. In the I2C master mode, the JY-901 acts as a master to the external sensors. The sensing module directly obtains data from the auxiliary sensors, allowing the on-chip DMP to generate sensor fusion data without intervention from the system processor. For example, In the I2C master mode, the module can be configureured to perform burst reads and returning the data from a magnetometer. While, in the pass-through mode, the module directly connects the primary and auxiliary I2C. When using JY-901 for 9-axis Sensor Fusion using I2C Interface, the system processor is an I2C master to the module.

JY-901 module also features the sensor data registers and the FIFO. The data registers contain the latest gyro, accelerometer, auxiliary sensor and temperature measurement data. They are read-only registers and are accessed via the serial interface. Data from these registers may be read anytime. The interrupt function 
may be used to determine when new data is available. The JY-901 contains a 1024-byte FIFO register that is accessible via the serial interface. The FIFO conFigureuration register determines which data is to be written into the FIFO. Possible choices include gyro data, accelerometer data, temperature readings, auxiliary sensor readings etc.

For the JY-901, accelerometer measurements are passed through a conFigureurable Digital High Pass Filter (DHPF) in order to eliminate bias due to the gravity. A qualifying motion sample is one where the high passed sample from any axis has an absolute value exceeding a user-programmable threshold. A counter increment for each qualifying sample and decrements for each non-qualifying sample. Once the counter reaches a userdefined counter threshold, a motion interrupt is triggered.

An on-chip temperature sensor and ADC are used to measure the chip die temperature. The readings from the ADC are read from the FIFO or the sensor data registers. The temperature sensor range is from -40 to $+85^{\circ} \mathrm{C}$.

\section{Measurement System Using JY-901 Module}

In this session, a dynamic motion monitoring system with JY-901 module is set up and tested (Figure 2 to Figure 4). The system consists of a JY-901 sensing module, a TTL (Time To Live)-USB converter and a control computer. The JY-901 module functions to monitor the dynamic behavior of the test structure by measuring its attitude angles (yaw, roll and pitch), acceleration motions and magnetic field data.

As shown in Figure 2 and 3, to connect the JY-901 to the TTLUSB converter, the four pins of the JY-901 are connected to the corresponding pins of the converter, whose other side is directly plugged in a USB port of the control computer. By a trigger from the computer, the data measured by the JY-901 are read from the serial port and saved with time stamps by the computer. In this setup, no expensive and cumbersome oscilloscope or signal amplifiers are included. In addition, the JY-901 module is powered by the computer so no extra power supply is needed. The elimination of oscilloscope, amplifiers and power unit greatly simplifies the measurement system. As a result, the system becomes portable and convenient, especially suitable for onsite measurement where the available space is limited.

\section{GY953 9-Axis Sensing Module}

AHRS (Attitude and Heading Reference System) is widely used in projects requiring real-time three dimensional tracking such as robots, autonomous vehicles, image stabilization systems and head-tracking (Chee et al. 2013). It integrates gyroscopes and fuses this data with accelerometer data and magnetometer data. With sensor fusion, drift from the gyroscope integration is compensated for by the reference vectors, namely the gravity and the earth magnetic field. The drift-free orientation makes the AHRS a more cost-effective solution than the conventional high-grade IMUs (Inertial Measurement Units) that only integrate gyroscopes and rely on a very high bias-stability of the gyroscopes.
GY953 AHRS module is a small sensor module consists of 9 degrees of freedom motion sensors ( 3 accelerometers, 3 gyroscopes and 3 magnetometers). With the size of $15 \mathrm{~mm} \times$ $15 \mathrm{~mm} \times 3 \mathrm{~mm}$, it provides real-time static and dynamic attitude angles optimized by Kalman Filter. Besides the bias tracking algorithm to eliminate the drift, GY953 also features a fuzzy algorithm to reduce the linear acceleration impact on the attitude calculation. Due to these characteristics, GY953 is applied in human motion capture, car navigation, platform stabilization, consumer electronics, monitoring device etc. It is especially suitable for applications which have critical demand on size and power consumption. From the control computer, GY953 can direct output Euler angles (yaw, roll, pitch) and 9-axis sensor raw data from its accelerometers, gyroscopes and magnetometers. Figure 5 shows the picture of GY953 sensing module.

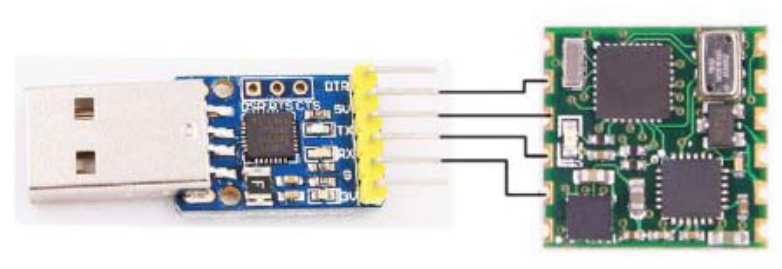

Figure 2: JY-901 module connected to TTL-USB converter

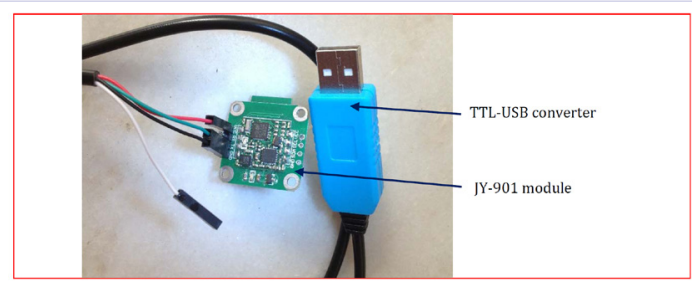

Figure 3: JY-901 module with TTL-USB converter

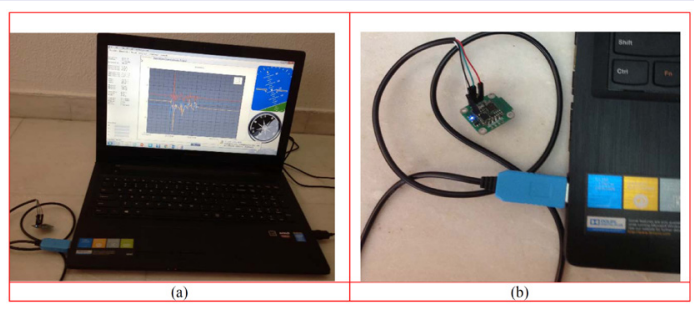

Figure 4: 9-axis motion monitoring system with JY-901 sensing module

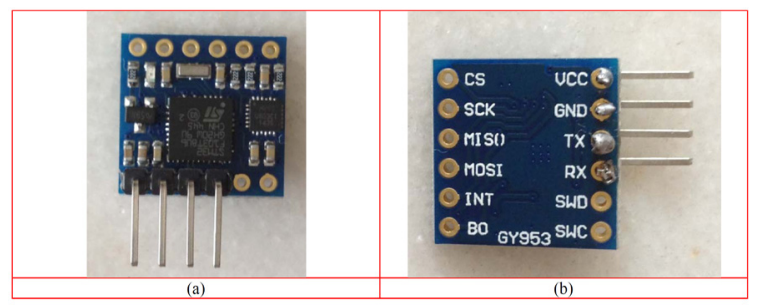

Figure 5: GY953 sensing module 


\section{GY953 Technical Specifications}

GY-953 module has an MPU-925 as its 9-axis motion tracking chip, which is designed for battery operated, high performance electronics products. GY953 combines an on-board Digital Motion Processor to process the complex motion fusion algorithms from the gyroscopes, accelerometer and compass. In the past few years, 9-axis motion tracking has become a key function in many consumer electronics devices as it provides an intuitive way to interact with these devices.

GY953 employs the STM32 32-bit flash microcontroller as its core processor. It offers high performance, low power operation, digital signal processing, while maintaining full integration and ease of development. The microcontroller offers two 12-bit ADCs, three general purpose 16-bit timers plus one PWM timer, as well as standard and advanced communication interfaces. A comprehensive set of power-saving modes caters for low-power applications. The working temperature range is from -40 to +85 ${ }^{\circ} \mathrm{C}$.

\section{Measurement System Using GY953 Module}

To test the performance of the GY953 motion sensing system, a bendable triangle frame is used as the test structure. The frame is made of iron with dimensions of $30 \mathrm{~cm} \times 13 \mathrm{~cm} \times 4.2 \mathrm{~cm}$. Before measurement, the GY953 module is mounted to the structure under testing and the module is connected to the control computer via the TTL-USB converter (Figure 6-8). As the host structure moves, the 3-axis gyroscope, the 3-axis accelerometer and the 3-axis compass in the GY953 module tracks the dynamic motion of the structure. The onboard Digital Motion Processor of the GY953 processes the complex motion fusion algorithms and provides real-time attitude and acceleration data. The processed signals are then sent to the computer via the TTL-USB converter.

Figure 9 plots the measured attitude angles from the GY953 module. Examination of the curves illustrate that the module captures the bending of the slant beam in roll direction. As the slant beam moves, there exist motions introduced by hands in pitch and yaw directions. However, it can be seen from Figure 9 that such motion is small compared to the movement in roll direction.

As the GY953 is powered by the computer, the elimination of the power unit and oscilloscope reduces the complexity of the dynamic monitoring system, making measurement convenient and easy. As the system is simplified, the deployment fee and the electrical insulation cost on-site are also reduced.

\section{Verification Experiments}

In this section, experiments are carried out to monitor the motion of a sample host structure. The performance of the JY-901 system and the GY953 system is experimentally investigated. As shown in Figure 10, the JY-901 and the GY953 modules are fixed to the movable slant beam side by side using glue tape. In such setup, the bending input is the same for both sensing systems.

The slant beam of the triangle frame is moved to produce a bending in the pitch direction. Upon the trigger from the same

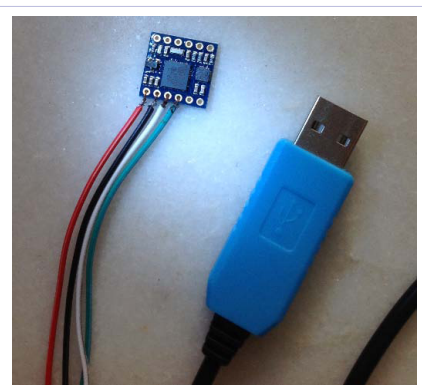

Figure 6: GY953 sensing module with TTL-USB converter

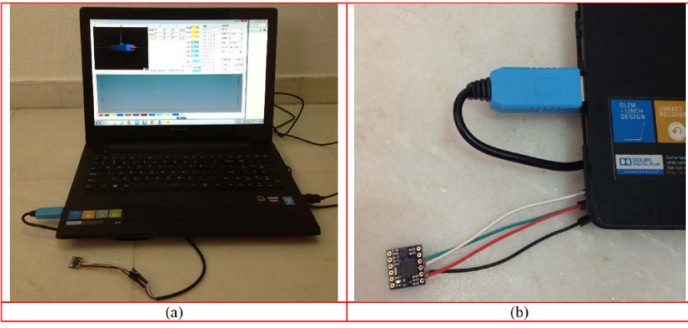

Figure 7: 9-axis motion measurement system with GY953 module

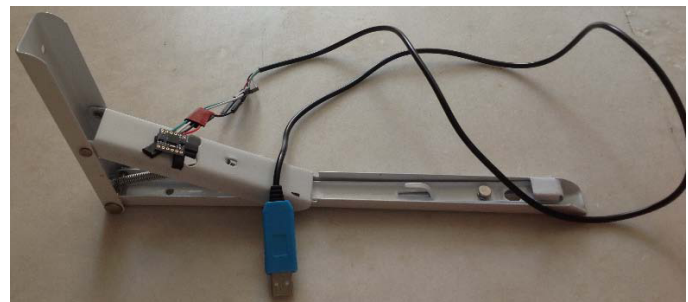

Figure 8: Experimental setup to monitor the motion of a triangular frame by GY953module

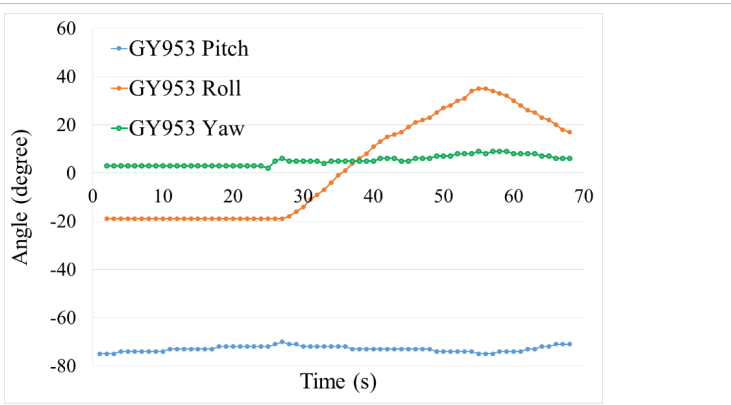

Figure 9: Angles of the triangular frame measured by GY953 module

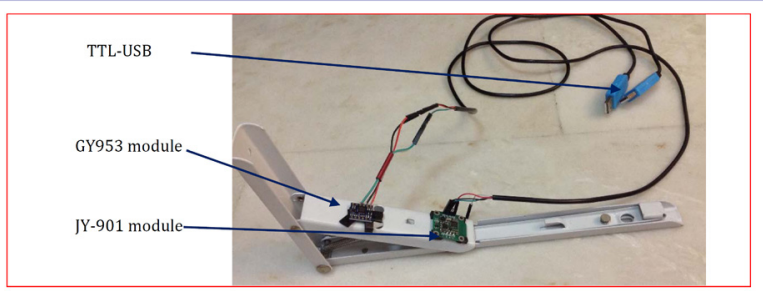

Figure 10: Experimental setup to measure the bending angles of the triangular frame 
control computer, the JY-901 and the GY953 sensing modules start to catch the motion of the movable beam. The on-chip DMP processes the measured data to compensate the drift using the acceleration and the magnetic field data as reference. After that, the processed data is transmitted to the computer for data logging and analysis.

Figure 11 shows the measured attitude angle data from the JY-901 and the GY953 systems. Examination of the curves illustrates that the two sets of pitch angles have good agreement with each other. They demonstrate matching cycle of signals and describe the input bending with peak and trough at the same time instants. The angle difference between the two sets of curves is within $0.2 \%$, well within the range normally expected in experimental analysis.

\section{Wireless Sensing System}

To build the wireless sensing system, HC-12 Wireless Serial Port Communication Module is incorporated into the measurement system. HC-12 module is a new-generation wireless data transmission module. Its working frequency band is $433.4 \mathrm{MHz}$ to $473.0 \mathrm{MHz}$. Multiple channels can be set with the stepping of $400 \mathrm{KHz}$ within this range. The maximum transmitting power is $100 \mathrm{~mW}(20 \mathrm{dBm})$ and the communication distance is $1000 \mathrm{~m}$ in open space. The module has a small footprint of $27.8 \mathrm{~mm} \times 14.4 \mathrm{~mm} \times 4 \mathrm{~mm}$. Consequently it is convenient for customers to include this module into their application systems. It has a PCB antenna on the module pedestal and an antenna socket for external spring antenna.

As illustrated in Figure 12, the base station of the measurement system consists of an HC-12 wireless communication module and a TTL-USB converter. The base station serves to transmit commands, trigger the sensing motes and receive the measured data. Figure 13 shows the picture of one sensing mote. It consists of a JY-901 sensing module, an HC-12 wireless module and a 3.7V battery pack. The Lithium Polymer battery is used to power the sensing module as well as the wireless module. The battery is a single-cell and has capacity of $1200 \mathrm{mAh}$. By using the ON/ OFF switch on the battery pack, the sensing mote can be turned off to save battery when necessary.

When the battery pack is switched on, the JY-901 module starts measuring three attitude angles, three acceleration motions and three magnetic field data. The measured data are then wirelessly transmitted via the HC-12 module to the control computer. Figure 14 and 15 show the translational acceleration data and the rotational attitude angles measured by the wireless sensing mote. The triangle frame in Figure 10 is used as the test structure here. Trough experiments, it has been verified that the wireless transmission distance of the HC-12 can reach more than 60 meters and the data quality maintains the same even after passing through a few concrete walls.

\section{Conclusions}

In this project, two measurement systems are developed to detect the attitude angles and the accelerations to monitor the dynamic behavior of structures. JY-901 and GY953 modules are employed respectively in the systems as core sensing elements. The measured motion data give us insight into how the complex structures works. The data also provide information for the design optimization of the host structures. For the JY-901 system, besides the wired version in which the module is connected to the control computer via a USB cable, a wireless transmission version using HC-12 wireless unit is also built and tested. From

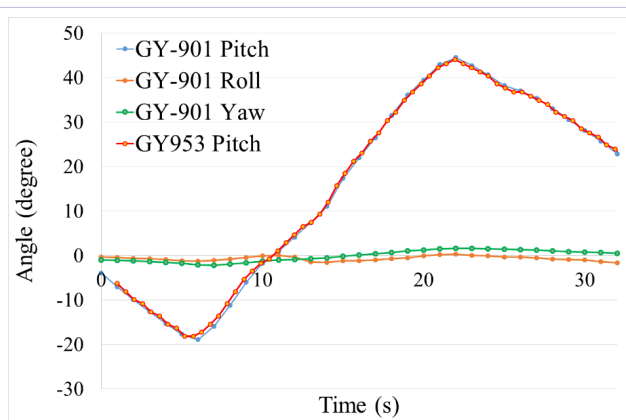

Figure 11: Measured angles of the triangular frame by JY-901 and GY953 modules

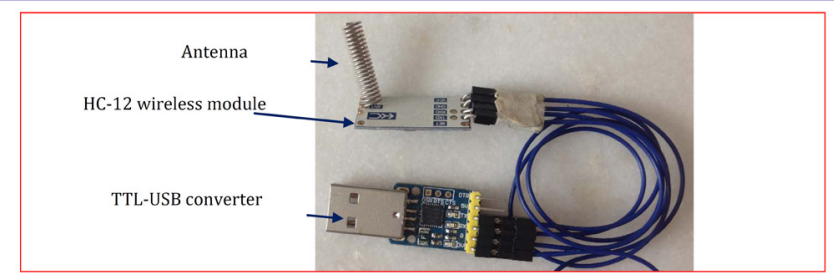

Figure 12: Base station for JY-901 (HC-12 wireless transmission module with TTL-USB converter)

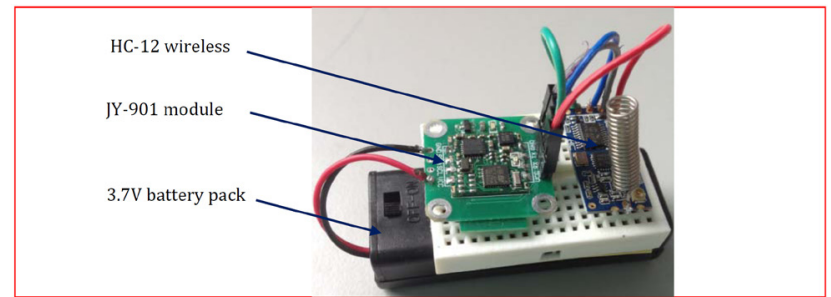

Figure 13: Wireless sensing mote of JY-901 with HC-12 wireless transmission module

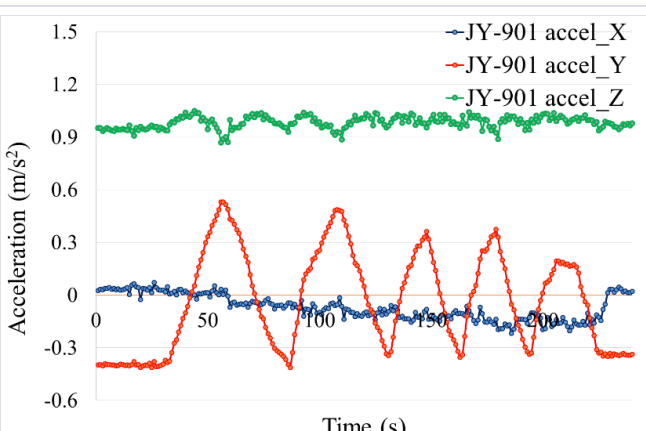

Figure 14: Acceleration of the triangular frame measured by JY-901 wireless mote 


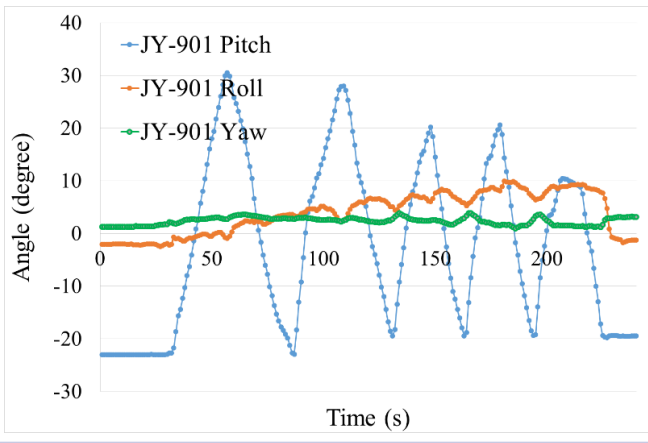

Figure 15: Angles of the triangular frame measured by JY-901 wireless mote

the experimental results, it is concluded that the systems can accurately tract the movement of the test structures.

\section{Acknowledgments}

The authors thank the National Research Foundation, Keppel Corporation and National University of Singapore for supporting this work done in the Keppel-NUS Corporate Laboratory. The conclusions put forward reflect the views of the authors alone, and not necessarily those of the institutions within the Corporate Laboratory.

\section{References}

1. Frangopol DM, Soliman M. Life-cycle of structural systems: recent achievements and future directions. Structure and Infrastructure Engineering: Maintenance, Management, Life-Cycle Design and Performance. 2015;12(1):1-20.

2. Jun Yue Robot Control. JY-901 Inertial Navigation Module Manual. 2015:1-2.

3. Chee KY, Zhong ZW. Control, navigation and collision avoidance for an unmanned aerial vehicle. Sensors and Actuators A: Physical. 2013;190:66-76.

4. Kongsberg Maritime. Motion and heading sensor products. 2016.

5. Brownjohn MW, Stefano AD, Xu YL, Wenzel H, Aktan AE. Vibrationbased monitoring of civil infrastructure: challenges and successes. Journal of Civil Structural Health Monitoring. 2011;1(3):79-95.

6. Ren Q, Wang B, Deng Z, Fu M. A multi-position self-calibration method for dual-axis rotational inertial navigation system. Sensors and Actuators A: Physical. 2014;219:24-31.

7. Yuan Q, Chen I. Localization and velocity tracking of human via 3 IMU sensors. Sensors and Actuators A: Physical, 2014;212:25-33.

8. Zhu XQ, Law S S. Recent developments in inverse problems of vehicle-bridge interaction dynamics. Journal of Civil Structural Health Monitoring. 2016;6(1):107-128. 